\title{
Numerical investigation of microbending loss in optical fibres
}

\author{
X.Q. Jin and F.P. Payne
}

\begin{abstract}
Microbending plays a key role in the bend loss of optical fibres. To numerically investigate microbending induced loss, an analytical model for microbending in optical fibres with arbitrary refractive index profiles is presented. In this model, random perturbations of the fibre core along the fibre axis are described by an analytical function whose power spectral density is derived from an exponential autocorrelation function. Using the model together with the beam propagation method, microbending loss is investigated for several different types of optical fibre, which include the traditional single-mode/multimode fibres (SMF/MMF) as used in existing optical networks, and typical few-mode/ring-core fibres (FMF/RCF) with the potential for future ultra high-speed optical networks. The validity of the proposed model is demonstrated by comparing the microbending loss of a SMF computed using coupled mode theory and our results. Simulation results show that, under the condition of launching only the fundamental mode into the optical fibres, the SMF and RCF supporting only one radial mode have nearly equal microbending loss, whilst the FMF and MMF have relatively low microbending loss. In addition, the microbending loss of the RCF is shown to be dependent on the ring core thickness.
\end{abstract}

Index Terms-Beam propagation method, Fiber optics systems, Microbending loss, Optical fibre.

\section{INTRODUCTION}

$\mathrm{T}$ he low attenuation of optical fibres has made an important contribution to the fast growth of optical fibre based networks for high-speed communications in the last few decades. Apart from the inherent low loss of optical fibres, bending an optical fibre from a straight axis is one of key extrinsic effects increasing the attenuation of an optical fibre. Over the last 40 years, extensive investigations have been given to two mechanisms of bending that causes the escape of light from an optical fibre: macrobending and microbending [1-9]. Macrobending usually results from the bending of an optical fibre at a relatively large radius. Macrobending induced loss has been well studied and understood [6-9] so that both analytical and numerical models are able to predict macrobending loss with good agreement with experiment [7,8]. Compared with macrobending, microbending occurs in practice when the bending radius is smaller than $1 \mathrm{~mm}$, because microbending

Manuscript received August 19, 2015. This work was supported by the U.K. Engineering and Physical Sciences Research Council under grant number EP/J008745/1.

X.Q. Jin and F.P. Payne are with the Department of Engineering Science, University of Oxford, Oxford, OX1 3PJ, U.K. (e-mail: xianqing.jin@eng.ox.ac.uk, frank.payne@lincoln.ox.ac.uk). results from the high-frequency random perturbations of the fibre core along the fibre axis [1-6]. The physical cause of the random perturbations is usually due to lateral contact of the optical fibre with rough surfaces and imperfect optical fibre fabrication processing. For an optical fibre with microbending, there is mode coupling between the guided modes and the cladding modes. The loss arises from the heavily attenuated power of the cladding modes due to the lossy plastic jacket surrounding the optical fibre.

The theoretical study on microbending induced loss in the traditional single-mode fibre (SMF) and multimode fibre (MMF) was carried out in 1970s, when analytical expressions for the microbending loss of an optical fibre were given by various researchers [2-5]. However, most analysis of microbending loss is usually based on the microbending distorted index distribution assumed to be expressed as a first order Taylor expansion [2-4]. To improve the accuracy of estimates of microbending loss, especially for complex optical fibres, a more precise model of microbending is required. Numerical solution of microbending loss is a good way of estimating the microbending loss of optical fibres with arbitrary refractive index profiles. Several numerical investigations of microbending loss have been reported [10-12], which, however, only considered an optical fibre with microbends at a constant bending radius. In practice, since the random perturbations of an optical fibre with microbending contain high-frequency components, a more precise model of microbending is particularly important.

In this paper, an analytical model for the microbending in optical fibres is proposed for the study of microbending loss. In the model, random perturbations of the fibre core along the fibre axis are described by an analytical function with a power spectral density derived from an exponential autocorrelation function. Using this model together with the beam propagation method, investigation is made of microbending loss of four different types of optical fibre. A widely used analytical expression for the microbending loss of a SMF was given by Marcuse [3] and experimentally proved [4, 13]. The validity of our model is verified by comparing the microbending loss for a SMF with Marcuse's analytical results. Recent intensive research on space division multiplexing (SDM) for overcoming the capacity bottleneck imposed by the nonlinear Shannon limit in SMFs $[14,15]$ has resulted in new types of optical fibre to meet the requirements of SDM technology [16-20]. As well as the traditional SMF/MMF [21], special consideration is given in 


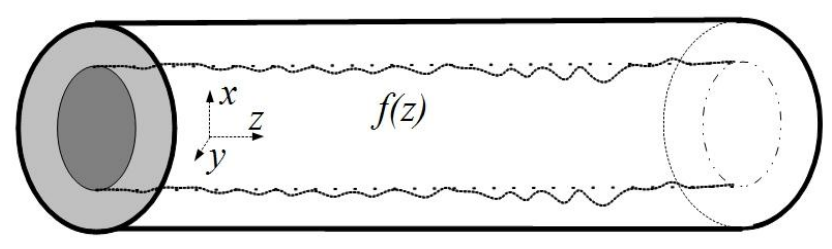

Fig. 1. Random perturbations of the fibre core.

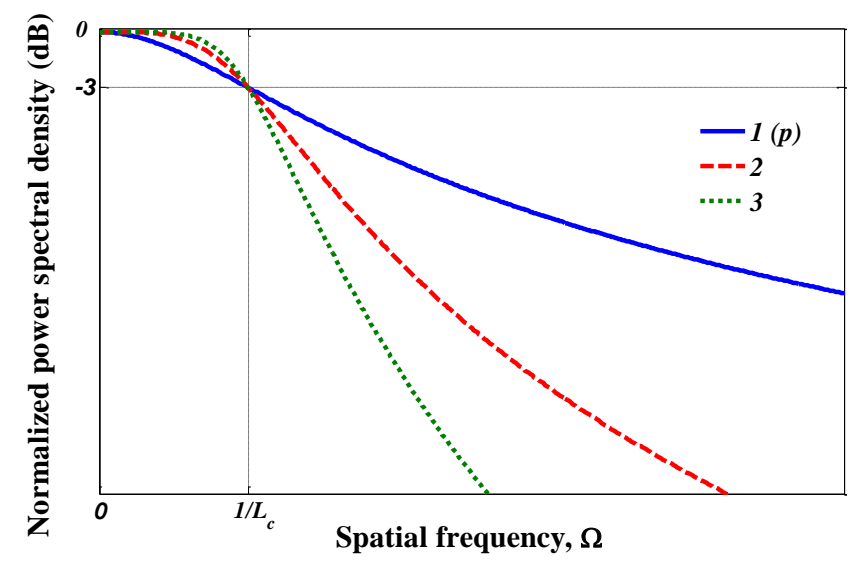

Fig. 2. Power spectral density of autocorrelation functions with typical values of $p(1,2,3)$.

this paper to the microbending loss of few-mode fibres (FMFs) $[16,17]$ and ring-core fibres (RCFs) [18-20] which can be potentially used for future ultra high-speed optical networks.

\section{MODELLING OF MICROBENDING IN OPTICAL FIBRES}

In this section, an analytical function is derived for modeling microbending in optical fibres with arbitrary refractive index profiles. For an ideal optical fibre, the fibre core is perfectly straight. However, in practice, the fibre core randomly deviates from an ideal straight line, which results in the corresponding variation of the whole core layer of the fibre as shown in Fig. 1. Such deviation of the fibre core can be described by a random function, $\vec{f}(z)[2,3]$.

$$
\vec{f}(z)=f_{x}(z) \vec{x}+f_{y}(z) \vec{y}
$$

where $f_{x}$ and $f_{y}$ are x- and y-components of $\vec{f}(z)$, respectively. For simplicity, only the x-component $f_{x}$ is considered here, and $\vec{f}(z)$ can be replaced by a scalar $f(z)$. Assuming that $f(z)$ is a stationary random function, the statistics of $f(z)$ are independent of the position $z$ along the fibre. For a long optical fibre consisting of a number of fibre sections, ensemble averages $\langle f(z)\rangle,\left\langle f(z)^{2}\right\rangle$ and the autocorrelation function $R(u)$ of $f(z)$ are the same at different fibre sections. Although $f(z)$ is a random function, the microbending due to random perturbations of the fibre core can be modelled by these three quantities.

As the fibre core varies around a straight line, the ensemble average $\langle f(z)\rangle$ is assumed to be zero. For stationary random process, the ensemble average is the same as space average. It indicates that the ensemble average $\left\langle f(z)^{2}\right\rangle$ can be obtained by measuring the square of the standard deviation, $\sigma^{2}$, of $f(z)$ at a number of $z$ positions along an optical fibre. From the basic relation between the ensemble average $\left\langle f(z)^{2}\right\rangle$ and the autocorrelation function $R(u),\left\langle f(z)^{2}\right\rangle$ (or $\sigma^{2}$ ) is equal to $R(0)$. Typically, the autocorrelation function $R(u)$ decreases with increasing $u$. The length scale over which $R(u)$ decreases is normally used to describe how much $f(z)$ is correlated at two different $z$ positions. This length scale is known as the correlation length, which will be discussed in the following. Considering that the autocorrelation function $R(u)$ is related to the spatial frequencies contained in the random function $f(z)$, the Fourier transform of $R(u)$ and $f(z)$ are taken to obtain the power spectral density function $\tilde{R}(\Omega)$ and spectrum $\tilde{f}(\Omega)$.

$$
\begin{gathered}
\tilde{R}(\Omega)=\frac{1}{2 \pi} \int_{-\infty}^{\infty} e^{j \Omega u} R(u) d u \\
\tilde{f}(\Omega)=\lim _{L \rightarrow \infty} \int_{-L / 2}^{L / 2} e^{j \Omega z} f(z) d z
\end{gathered}
$$

The relation between $\tilde{R}(\Omega)$ and $\tilde{f}(\Omega)$ is given by,

$$
\tilde{R}(\Omega)=\lim _{L \rightarrow \infty} \frac{\left\langle|\tilde{f}(\Omega)|^{2}\right\rangle}{2 \pi L}
$$

where $L$ is the length of the fibre. From the above analysis, the random function $f(z)$ is assumed to be a sum of sinusoids of amplitude proportional to $\sqrt{\widetilde{R}(\Omega)}$, at multiples of a fundamental frequency $\omega_{0}$ and with random phases. This forms the basis of constructing a suitable analytical function to describe random perturbations of the fibre core. The random function $f(z)$ can be written as.

$$
f(z)=A \sum_{n=1}^{N} \sqrt{\tilde{R}\left(n \omega_{0}\right)} \cos \left(n \omega_{0} z+\varphi_{n}\right)
$$

where $A$ is a normalisation coefficient for a given value of $\sigma^{2}$, and $\varphi_{n}$ is a uniformly distributed random phase. Because the ensemble average of $f(z)$ is zero, the sum over $n$ must start at $n=$ 1.

As seen from Eq. (5), the random function $f(z)$ mainly depends on the power spectral density function $\tilde{R}(\Omega)$ of the autocorrelation function. A wide range of autocorrelation functions can be described by the following equation $[1,2,22]$.

$$
\begin{gathered}
\tilde{R}(\Omega)=\frac{C \sigma^{2}}{1+\left(\Omega L_{c}\right)^{2 p}} \\
C=\left[\int_{-\infty}^{\infty} \frac{1}{1+\left(\Omega L_{c}\right)^{2 p}} d \Omega\right]^{-1}
\end{gathered}
$$

where $L_{c}$ is correlation length. A typical value of $p$ is 1,2 , or 3 depending on the external stress on the fibre and fibre fabrication processing. The power spectral density with these typical values of $p$ is shown in Fig. 2. It is clear to see from Fig. 2 and Eq. (6) that the $3 \mathrm{~dB}$ point of the power spectral density is equal to the inverse of the correlation length $\left(1 / L_{c}\right)$. Here, the $p$ value is set to 1 to represent the power spectral density of an exponential autocorrelation function which is often used to model many random processes of waveguides [23]. The 
exponential autocorrelation function and its power spectral density function are given by.

$$
\begin{aligned}
& R(u)=\sigma^{2} \exp \left(\frac{-|u|}{L_{c}}\right) \\
& \tilde{R}(\Omega)=\frac{\sigma^{2} L_{c}}{\pi\left(1+\Omega^{2} L_{c}{ }^{2}\right)}
\end{aligned}
$$

Since the ensemble average $\left\langle f(z)^{2}\right\rangle$ is equal to $\sigma^{2}$, the coefficient $A$ in Eq. (5) can be derived as follows.

$$
<f(z)^{2}>=A^{2} \frac{L_{c} \sigma^{2}}{2 \pi} \sum_{n=1}^{N} \frac{1}{1+\left(n \omega_{0}\right)^{2} L_{c}{ }^{2}}=\sigma^{2}
$$

For a large number of small fundamental frequencies, Eq. (10) can be written as,

$$
\frac{<f(z)^{2}>}{\sigma^{2}} \approx A^{2} \frac{L_{c}}{2 \pi \omega_{0}} \int_{0}^{\infty} \frac{d \omega}{1+\omega^{2} L_{c}{ }^{2}}=\frac{A^{2}}{4 \omega_{0}} \approx 1
$$

With the calculated $A$ being approximately equal to $2 \sqrt{\omega_{0}}$ in Eq. (11), the new form of Eq. (5) can then be expressed as,

$$
f(z)=2 \sum_{n=1}^{N} \sqrt{\omega_{0} \tilde{R}\left(n \omega_{0}\right)} \cos \left(n \omega_{0} z+\varphi_{n}\right)
$$

The maximum frequency $\left(\Omega_{\max }=N \omega_{0}\right)$ in Eq. (12) is set by requiring that a fraction $(\eta<1)$ of the total power is correctly described by $\tilde{R}(\Omega)$, so that

$$
\int_{-\Omega_{\max }}^{\Omega_{\max }} \tilde{R}(\Omega) d \Omega=\eta \sigma^{2}
$$

Substitute Eq. (9) into (13),

$$
\Omega_{\max }=\frac{1}{L_{c}} \tan \left(\frac{\eta \pi}{2}\right)
$$

The value of $\Omega_{\max }$ is then used to determine the number of sinusoids $(N)$ at a given fundamental frequency, $\omega_{0}$. Generally, $\omega_{0}$ is specified in order to fit the sinusoid of no more than one period at the fundamental frequency into an optical fibre of the whole length $L$ :

$$
\omega_{0} \leq \frac{2 \pi}{L}
$$

Therefore, the integer $N$ is obtained as ,

$$
N=\left\lceil\frac{\Omega_{\max }}{\omega_{0}}\right\rceil \geq \frac{L}{2 \pi L_{c}} \tan \left(\frac{\eta \pi}{2}\right)
$$

where ' \lceil\rceil ' represents the smallest integer not less than the number inside.

To be used in numerical analysis, the continuous function $f(z)$ must be discretised at a spatial interval, $\Delta z$. Similar to the Nyquist sampling theorem in the field of digital signal processing, the spatial interval is set so that the sampling frequency $2 \pi / \Delta z$ is greater than or equal to twice the maximum frequency $\left(2 \Omega_{\max }\right)$.

$$
\Delta z \leq \frac{\pi}{\Omega_{\max }}=\pi L_{c}\left[\tan \left(\frac{\eta \pi}{2}\right)\right]^{-1}
$$

Using the above analytical functions in a BPM program, light propagation over an optical fibre with microbends can be simulated for the study of microbending loss in various types of optical fibre, which is discussed in the following.

\section{SimULATION PARAMETERS}

Having developed an analytical model for microbending in optical fibres, in this section we discuss the choice of parameters that are used in the numerical simulations. As a practical compromise we choose $\eta=0.99$ in Eq. (13), which means that $99 \%$ of the power described by $\widetilde{R}(\Omega)$ is accounted for. The fundamental frequency $\omega_{0}$ is chosen to be $2 \pi / L$. From Eq. (16), the number of sinusoids $N$ is approximately equal to $64 L / 2 \pi L_{c}$. The spatial interval $\Delta z$ is fixed at $1 \mu \mathrm{m}$, which is smaller than the maximum allowable value of $\pi L_{c} / 64$ for the values of $L_{c}$ in the range of $\mathrm{mm}$.

To simulate the light propagation in optical fibres, the finite-difference beam propagation method (FD-BPM) with the Crank-Nicolson method is adopted [24]. The FD-BPM is based on the solution of the Helmholtz equation in the paraxial approximation.

$$
\left.\frac{\partial E}{\partial z}=\frac{1}{2 j n_{a} k_{0}}\left[\frac{\partial^{2} E}{\partial x^{2}}+\frac{\partial^{2} E}{\partial y^{2}}+\left(n(x, y, z)^{2}-n_{a}{ }^{2}\right) k_{0}{ }^{2} E\right)\right]
$$

where $E(x, y, z)$ is the electric field, $n_{a}$ is an arbitrary refractive index which is normally the refractive index of the cladding layer, $k_{0}$ is the wavenumber in free space at a wavelength of $1550 \mathrm{~nm}, n(x, y, z)$ is the refractive index profile of the optical fibre that can be written in terms of $f(z)$ in Eq. (12) and the ideal refractive index profile at the cross-section of the optical fibre, $n_{0}(x, y)$ as,

$$
n(x, y, z)=n_{0}(x-f(z), y)
$$

To prevent boundary reflections for the solution of Helmholtz equation in Eq. (18), a simple transparent boundary condition (TBC) is adopted which is based on the assumption that the field near the boundary acts as an outgoing plane wave [25]. The TBC is generally effective for the simulation of radiation loss by allowing radiation to escape from the computational domain.

With the FD-BPM and TBC, microbending loss of four typical optical fibres was obtained by monitoring the optical power variation along the propagation direction. Detailed parameters of these optical fibres are shown in Table I. The refractive index profile and amplitude of the fundamental mode are plotted in Figs. 3(a,b). The step-index SMF with a core diameter of $8.2 \mu \mathrm{m}$ [8] has the smallest effective area of the fundamental mode as shown in Fig. 3(c). To support more than one spatial mode, the core diameter of the FMF and MMF [17, 21] with parabolic index profiles and relatively large numerical aperture (NA) are $19.6 \mu \mathrm{m}$ and $50 \mu \mathrm{m}$, respectively. As seen in Fig. 3(b), the electric field of the fundamental mode of the FMF is slightly larger than the SMF, but smaller than the MMF. The RCF [20] has a parabolic index profile in the ring core layer with a ring radius of $10.5 \mu \mathrm{m}$ and a ring core thickness of $5.4 \mu \mathrm{m}$, which result in one radial mode and 7 azimuthal modes. The ring radius is defined as the radius of the maximum peak of the refractive index profile. The ring-shape fundamental mode in the RCF as shown in Fig. 3(d) has a large effective area compared with other three fibres. To reduce the computation demands at a small xy-mesh size of $0.15 \mu \mathrm{m}$, the cladding radius 


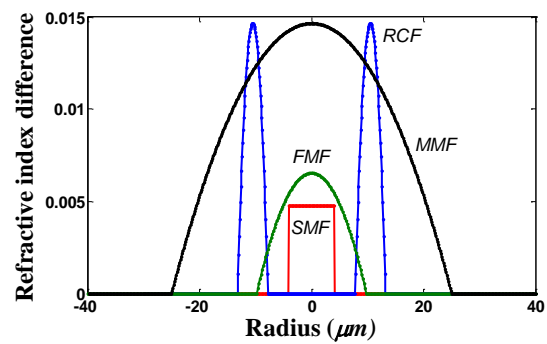

(a)

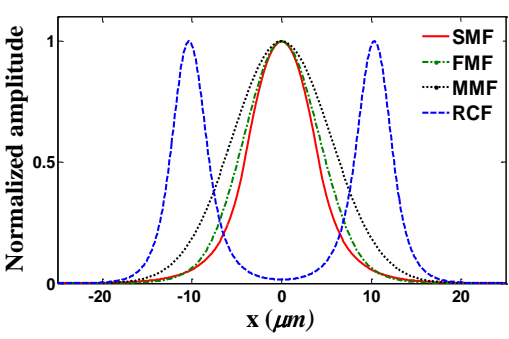

(b)

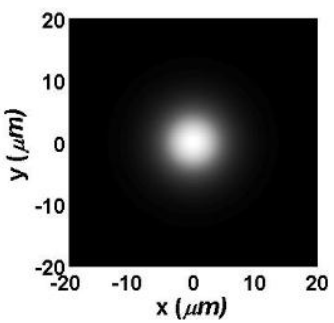

(c)

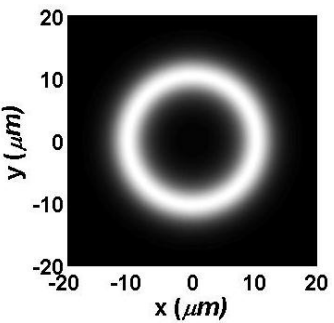

(d)

Fig. 3. (a) Refractive index profiles of the SMF, FMF, MMF and RCF. (b) Normalized amplitude of the fundamental modes. (c,d) Electric fields of the fundamental modes of the SMF (c) and the RCF (d).

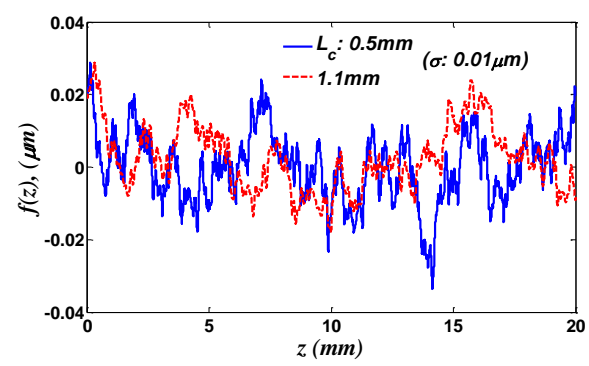

(a)

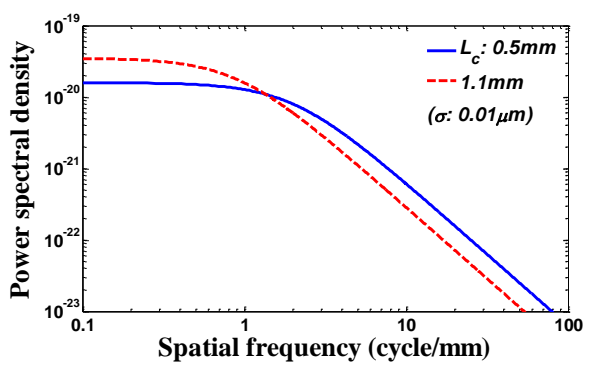

(b)

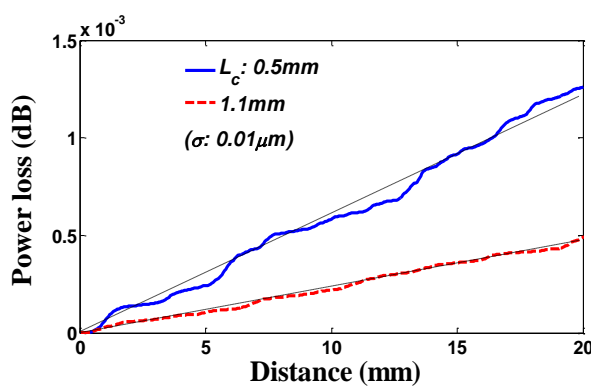

(c)

Fig. 4. (a) Variation of the fibre core as a function of propagation distance, $z$; (b) Power spectral density of an exponential autocorrelation function; (c) Power loss versus propagation distance $z$. $\left(L_{c}: 0.5 \mathrm{~mm}\right.$ or $\left.1.1 \mathrm{~mm}, \sigma: 0.01 \mu \mathrm{m}\right)$.

TABLE I

PARAMETERS OF OPTICAL FibRES

\begin{tabular}{lcccccc}
\hline $\begin{array}{l}\text { Optical } \\
\text { fibre }\end{array}$ & $\begin{array}{c}\text { Spatial } \\
\text { modes }\end{array}$ & $\boldsymbol{l}_{\max }{ }^{a}$ & $\boldsymbol{m}_{\max }{ }^{a}$ & $\begin{array}{c}\Delta \boldsymbol{n}_{\text {eff }}{ }^{b} \\
\left(\times \mathbf{1 0}^{-3}\right)\end{array}$ & $\begin{array}{c}\boldsymbol{A}_{\text {eff }}{ }^{2} \\
\left(\boldsymbol{\mu \boldsymbol { m } ^ { 2 } )}\right.\end{array}$ & $\boldsymbol{N A}$ \\
\hline $\boldsymbol{S M F}$ & 1 & 0 & 1 & 1.91 & 83.0 & 0.117 \\
$\boldsymbol{F M F}$ & 3 & 1 & 1 & 4.16 & 111.6 & 0.138 \\
$\boldsymbol{M M F}$ & 55 & 9 & 5 & 13.26 & 187.8 & 0.206 \\
$\boldsymbol{R C F}$ & 13 & 6 & 1 & 8.56 & 321.7 & 0.206 \\
\hline
\end{tabular}

${ }^{a} l_{\max }\left(m_{\max }\right)$ Maximum azimuthal (radial) mode index.

${ }^{b} \Delta n_{\text {eff }}$ : Minimum refractive index difference between the fundamental mode and cladding modes at a wavelength of $1550 \mathrm{~nm}$.

${ }^{c} A_{\text {eff }}$ : Effective area of the fundamental mode.

of SMF, FMF, MMF and RCF is $40 \mu \mathrm{m}, 40 \mu \mathrm{m}, 50 \mu \mathrm{m}$ and $40 \mu \mathrm{m}$, respectively. The length of the optical fibres, $L$, is set to $40 L_{c}$ if $L_{c} \geq 0.5 \mathrm{~mm}$, or $20 \mathrm{~mm}$ if $L_{c}<0.5 \mathrm{~mm}$.

\section{MiCROBENDING LOSS OF OPTICAL FIBRES}

With the analytical model for microbending in optical fibres and simulation conditions described in the previous sections, numerical investigation was undertaken of the microbending loss of four different types of optical fibre. Since extensive research on the microbending loss of the SMF has been reported both in theory and experiment $[4,13]$, it is necessary to validate the proposed model for the microbending loss of the SMF with the analytical solution in [3]. For an accurate comparison of microbending loss, only the fundamental mode is launched into the optical fibre. Therefore, the loss in the following discussions only includes the microbending loss of the fundamental mode, although it is straightforward to extend the computations to higher order modes.

\section{A. Validation of the analytical model for the microbending loss in the SMF}

The SMF with a step-index profile in Fig. 3(a) is used to validate the analytical model of microbending discussed in Section II. As an example, Fig. 4(a) shows two random functions $f(z)$ for random perturbations of the fibre core constructed with the power spectral density of an exponential autocorrelation function with $\sigma=0.01 \mu \mathrm{m}$ and $L_{c}=$ $0.5 \mathrm{~mm}, 1.1 \mathrm{~mm}$ in Fig. 4(b). The power spectral density contains both the low-frequency components for macrobending and the high-frequency components for microbending. It is easy to see that the graph of $f(z)$ with short correlation length varies faster than that with long correlation length due to the increased power in the high-frequency range as seen in Fig. 4(b). In practice, the threshold between microbending and macrobending is about 1 cycle/mm. In this paper, special consideration is given to the case of $L_{c}<1 \mathrm{~mm}$ so that the term microbending loss instead of bend loss including both microbending and macrobend loss is used in the following discussions.

The fundamental mode of the SMF with the intensity 


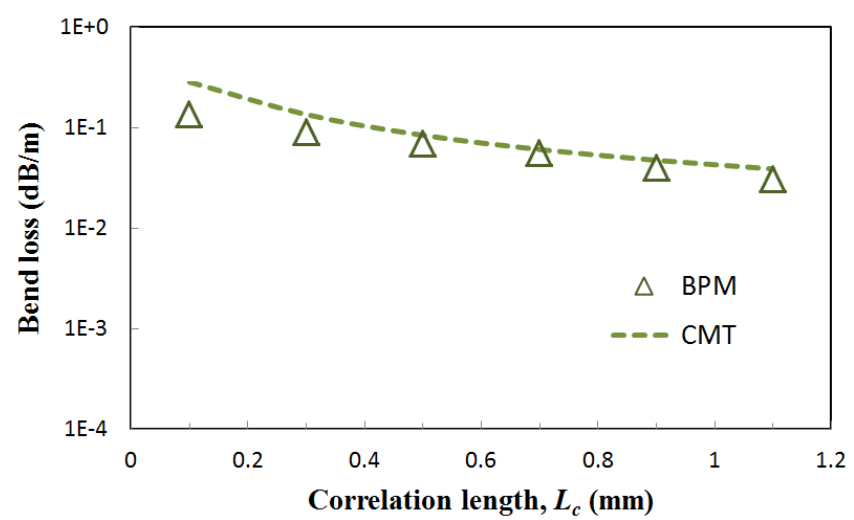

(a)

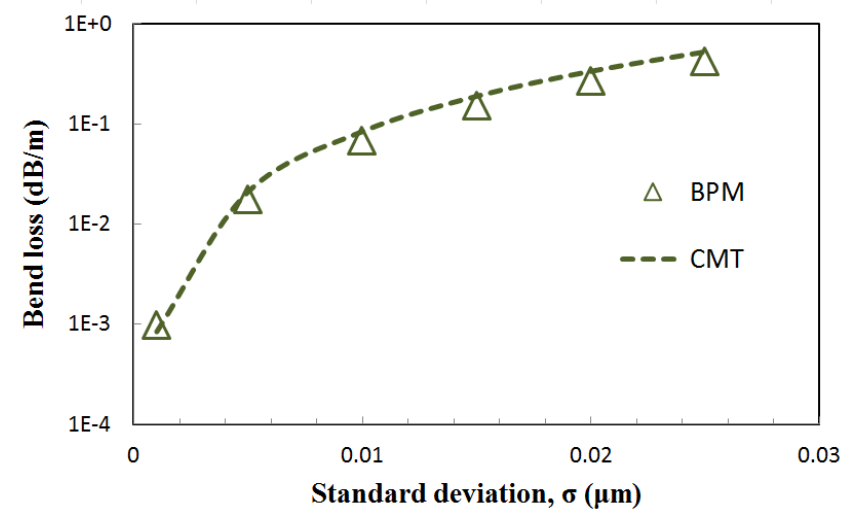

(b)

Fig. 5. Microbending loss of the SMF as a function of (a) correlation length ( $\sigma$ : $0.01 \mu \mathrm{m})$ and (b) standard deviation $\left(L_{c}: 0.5 \mathrm{~mm}\right)$.

distribution in Fig. 3(c) is used as an initial input field for the BPM simulation. As optical power can be recorded at each simulation step $\Delta z$, the graphs of calculated optical power loss against transmission distance are obtained in Fig. 4(c). The loss is equal to the slope of the curves of the power loss versus distance. As expected, the power loss for the short correlation length case is larger than that for the long correlation length case. The curves of the power loss slightly deviate from a straight line because the utilization of the simple transparent boundary condition may cause a very small amount of reflection [25]. However, such deviations do not affect the precision of the calculated microbending loss if the propagation distance is long enough.

To study the impact of the correlation length $L_{c}$ and standard deviation $\sigma$ on the microbending loss of the SMF, Figs. 5(a,b) show the microbending loss of the SMF against the correlation length $(\sigma=0.01 \mu \mathrm{m})$ and standard deviation $\left(L_{c}=0.5 \mathrm{~mm}\right)$. The microbending loss decreases with increasing the correlation length or decreasing the standard deviation. The calculated microbending loss based on the proposed method agrees very well with the results obtained from the analytical solution using coupled mode theory (CMT) [3]. This confirms the validity of the proposed analytical model for the study of microbending in optical fibres.

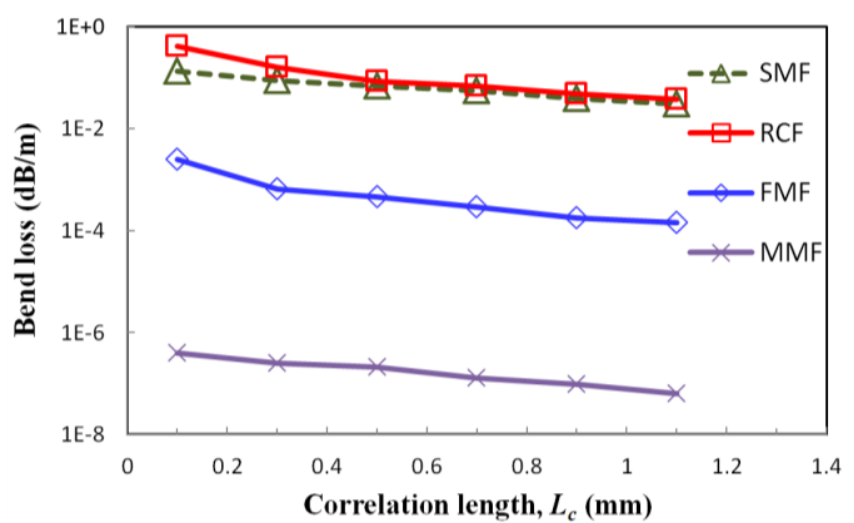

(a)

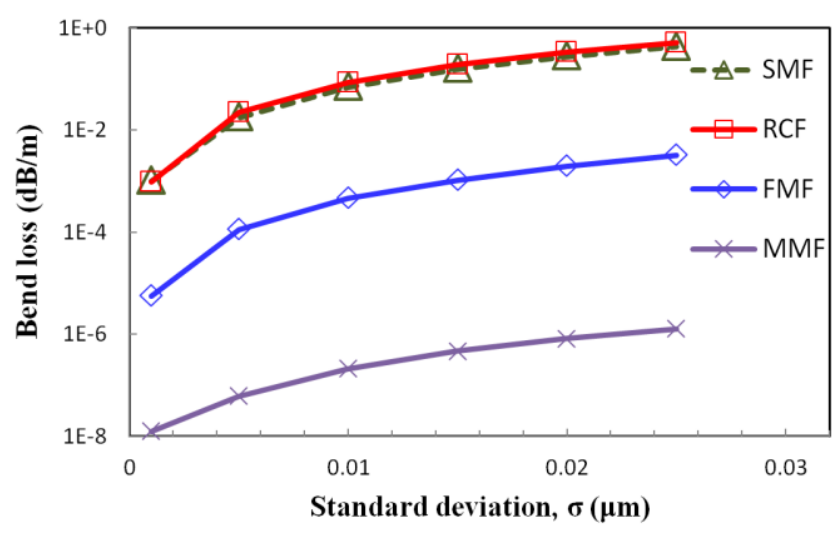

(b)

Fig. 6. Microbending loss of the SMF/FMF/MMF/RCF versus (a) correlation length $(\sigma: 0.01 \mu \mathrm{m})$ and (b) standard deviation $\left(L_{c}: 0.5 \mathrm{~mm}\right)$.

\section{B. Microbending loss of $F M F / M M F / R C F$}

After successfully verifying the validity of the proposed model for the microbending in the SMF, in this section, we investigate the microbending loss of the traditional MMF [21] and FMF/RCF [17, 20] for future ultra high-speed optical networks. The microbending loss of FMF/MMF/RCF is calculated with the recorded total optical power at each $\Delta z$ in the same way as mentioned in Section IV. A. Since most optical power remains in the launched fundamental mode for a short-distance transmission, the influence of mode coupling between the fundamental mode and other guided modes on the microbending loss is negligible. As shown in Figs. 6(a, b), the curves of the microbending loss for the FMF/MMF/RCF are parallel to the curve for the SMF. The microbending loss of the FMF (MMF) is about two (five) orders of magnitude lower than the SMF, because the minimum refractive index difference between the fundamental mode and cladding modes, $\Delta n_{\text {eff }}$ for the FMF (MMF) is much larger than that of the SMF. However, for the RCF which has the largest effective area of the fundamental mode and a $\Delta n_{\text {eff }}$ larger than the SMF, the microbending loss is nearly equal to the SMF. This can be explained by the fact that 1 ) The ring core thickness of the RCF determining the number of radial modes is almost equal to the radius of the SMF as seen in Fig. 3(a); 2) Both types of optical 


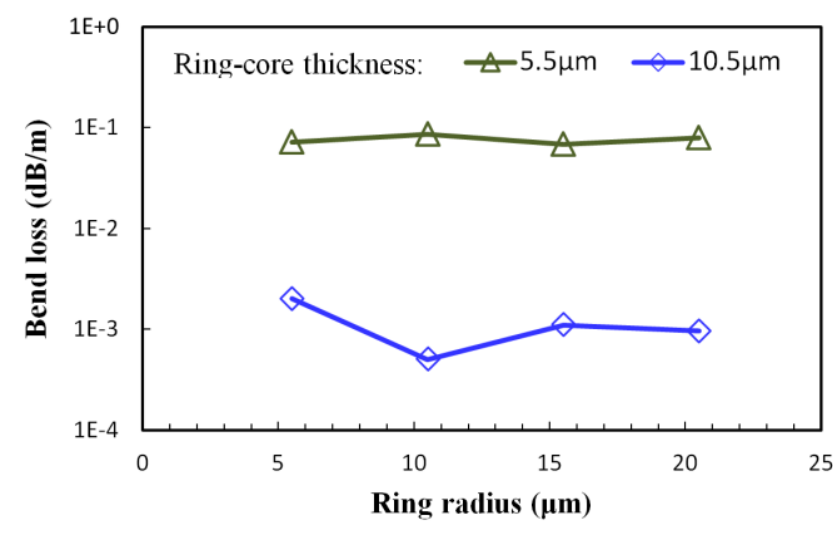

Fig. 7. Microbending loss of RCFs as a function of ring radius. Ring core thickness for one and two guided radial mode(s) $(m \leq 1$ and $m \leq 2)$ is $5.5 \mu m$ and $10.5 \mu \mathrm{m}$, respectively.

fibre only support one radial mode; 3) Compared with the SMF, the mode fields in the RCF are localised in the ring core layer rather than the centre of the fibre, which may result in increased interaction with the cladding modes.

It is well known that, from Marcuse's coupled mode theory, mode coupling between optical modes is determined by the power spectral density of the autocorrelation function, effective index (or propagation constant) difference between two different optical modes, refractive index profile and mode field distribution [2-3]. To gain a better understanding of the results of the RCF, the impact of the ring radius/thickness on the microbending loss due to the mode coupling between the guided modes and cladding modes is shown in Fig. 7, where the curves of the microbending loss versus the ring radius are plotted ( $\sigma=0.01 \mu \mathrm{m}$ and $L_{c}=0.5 \mathrm{~mm}$ ). The ring core thickness of the RCF supporting one and two guided radial mode(s) (radial index of guided modes: $m \leq 1$ and $m \leq 2$ ) is $5.5 \mu m$ and $10.5 \mu m$, respectively. It is interesting to note from Fig. 7 that the ring radius does not significantly affect the microbending loss, whilst RCFs with large ring core thickness that support 2 radial modes have relatively low microbending loss. This suggests that the microbending loss of the RCF is much more dependent on the ring core thickness. The main physical reason for the improved microbending loss is because the increase in the ring core thickness of the RCF results in a significantly increased effective index difference between the fundamental mode and cladding modes.

\section{CONCLUSIONS}

An analytical model for microbending in optical fibres with arbitrary refractive index profiles has been presented and numerical investigation of microbending induced loss has been computed using the FD-BPM method. The validity of the model has been successfully verified by good agreement between numerical and analytical values of the microbending loss for a SMF. Simulation results have shown that, for a fundamental mode launch condition, the SMF and RCF supporting only one radial mode have nearly equal microbending loss, whilst the
FMF and MMF have relatively low microbending loss. In addition, the microbending loss of the RCF is dependent on the ring core thickness.

\section{REFERENCES}

[1] W. B. Gardner, "Microbending Loss in Optical Fibers," Bell Syst. Tech. J., vol. 54, no. 2, pp. 457-465, Feb. 1975

[2] D. Marcuse, "Microbending Losses of Single-Mode, Step-Index and Multimode, Parabolic-Index fibers," Bell Syst. Tech. J., vol. 55, no. 7, pp. 937-955, Sep. 1976.

[3] D. Marcuse, "Microdeformation losses of single-mode fibers," Appl. Opt., vol. 23, no. 7, pp. 1082-1091, Apr. 1984.

[4] V. Arya, K. A. Murphy, A. Wang, and R. O. Claus, "Microbend losses in singlemode optical fibers: theoretical and experimental investigation," J. Light. Technol., vol. 13, no. 10, pp. 1998-2002, Oct. 1995.

[5] K. Petermann, "Microbending loss in monomode fibres," Electron. Lett., vol. 12, no. 4, pp. 107-109, Feb. 1976.

[6] J.H. Povlsen and S.B. Andreasen, "Analysis on splice, microbending, macrobending, and Rayleigh losses in $\mathrm{GeO}_{2}$-doped dispersion-shifted single-mode fibers," J. Light. Technol., vol. 4, no. 7, pp. 706-710, Jul. 1986

[7] D. Marcuse, "Curvature loss formula for optical fibers," J. Opt. Soc. Am., vol. 66, no. 3, pp. 216-220, Mar. 1976.

[8] R. T. Schermer and J. H. Cole, "Improved bend loss formula verified for optical fiber by simulation and experiment," IEEE J. Quantum Electron., vol. 43, no. 10, pp. 899-909, Oct. 2007.

[9] C. Schulze, A. Lorenz, D. Flamm, A. Hartung, S. Schroter, H. Bartelt, and M. Duparre, "Mode resolved bend loss in few-mode optical fibers," Opt. Express, vol. 21, no. 3, pp. 3170-3181, Feb. 2013.

[10] S. L. Jones, G. Murtaza, J. M. Senior, and N. Haigh, "Single-mode optical fiber microbend loss modeling using the finite difference beam propagation method," Opt. Fiber Technol., vol. 4, no. 4, pp. 471-479, Mar. 1998.

[11] Y. Yamauchi, M. Ikegaya, and H. Nakano, "Analysis of bent step-index optical fibres by the beam propagation method," IEE Proc. J Optoelectron., vol. 139, no. 3, pp. 201-207, Jun. 1992

[12] H. Deng, G. H. Jin, J. Harari, J. P. Vilcot, and D. Decoster, "Investigation of 3-D semivectorial finite-difference beam propagation method for bent waveguides," J. Light. Technol., vol. 16, no. 5, pp. 915-922, May 1998

[13] C. B. Probst, A. Bjarklev, and S.B. Andreasen, "Experimental verification of microbending theory using mode coupling to discrete cladding modes," J. Light. Technol., vol. 7, no. 1, pp. 55-61, Jan. 1989.

[14] D.J. Richardson, J. M. Fini, and L. E. Nelson, "Space-division multiplexing in optical fibres," Nat. Photonics, vol. 7, pp. 354-362, May 2013

[15] P.J. Winzer, 'Scaling optical fiber networks: challenges and solutions,' Optics \& Photon. News, Mar. 2015

[16] Y. Chen, A. Lobato, Y. Jung, H. Chen, V.A.J.M. Sleiffer, M. Kuschnerov, N.K. Fontaine, R. Ryf, D.J. Richardson, B. Lankl, and N. Hanik, "41.6 $\mathrm{Tb} / \mathrm{s}$ C-band SDM OFDM Transmission through 12 Spatial and Polarization Modes over 74.17 km Few Mode Fiber," vol. 33, no. 7, pp. 1440-1444, Apr. 2015

[17] S.R. Bickham, M.-J. Li, D. A. Nolan and J. Wang, "Few mode optical fibers for mode division multiplexing," US patent, US 2013/0071114 A1, Mar. 2013

[18] N. Bozinovic, Y. Yue, Y. Ren, M. Tur, P. Kristensen, H. Huang, A.E. Willner, and S. Ramachandran, "Terabit-Scale Orbital Angular Momentum Mode Division Multiplexing in Fibers", Science, vol. 340, no. 6140, pp. 1545-1548, Jun. 2013

[19] X.Q. Jin, R. Li, D.C. O'Brien, and F.P. Payne, "Linearly Polarized Mode Division Multiplexed Transmission over Ring-Index Multimode Fibres", IEEE Summer Topicals, (USA, 2013), Paper TuC4.3, Jul. 2013

[20] F. Feng, G. S. D. Gordon, X. Q. Jin, D. C. O’Brien, F. P. Payne, Y. Jung, Q. Kang, J. K. Sahu, S. U. Alam, D. J. Richardson, and T. D. Wilkinson, "Experimental characterization of a graded-index ring-core fiber supporting 7 LP mode groups," OFC/NFOEC'2015, Paper Tu2D.3, Mar. 2015 
[21] P. Pepeljugoski, S. E. Golowich, A.J. Ritger, P. Kolesar, and A. Risteski, "Modeling and simulation of next-generation multimode fiber links," J. Light. Technol., vol. 21, no. 5, pp. 1242-1255, May 2003

[22] R. Olshansky, "Mode Coupling Effects in Graded-index Optical Fibers", Applied Optics, vol. 14, no. 4, pp. 935-945, April 1975

[23] F. Ladouceur, J.D. Love, T.J. Senden, "Effect of side wall roughness in buried channel waveguides," IEE Proc.-Optoelectron., vol.141, pp. 242 248, Aug. 1994

[24] R. Scarmozzino, A. Gopinath, R. Pregla, and S. Helfert, "Numerical techniques for modeling guided-wave photonic devices," IEEE J. Sel. Top. Quantum Electron., vol. 6, no. 1, pp. 150-162, Jan. 2000.

[25] G. R. Hadley, "Transparent boundary condition for the beam propagation method," IEEE J. Quantum Electron., vol. 28, no. 1, pp. 363-370, Jan. 1992

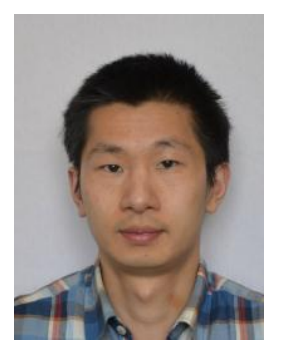

Xianqing Jin received the Ph.D. degree in optical communications in 2010 from Bangor University, UK. During his Ph.D. study, he focused on the field of optical OFDM for next generation local area networks and access networks, including the real-time DSP design/implementation for high-speed optical OFDM transmission. As a postdoctoral researcher at the University of Oxford in 2012-2015, he also studied the spatial multiplexing over ring-core fibres for future ultra-high-speed optical networks. $\mathrm{He}$ has been involved in three EU/UK-funded projects (ALPHA, OCEAN and COMIMO). His research interests cover a wide range of topics in optical OFDM, spatial multiplexing and real-time DSP for future optical communications. He has authored or co-authored $>60$ refereed papers, and holds 2 patents on implementation of core functions for high-speed optical OFDM systems.

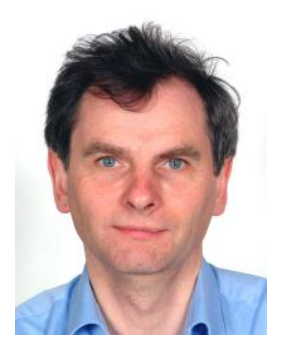

Professor Frank Payne is currently Reader Emeritus in Electronic Materials at Oxford University, and Fellow of Lincoln College Oxford. He has previously held senior appointments in the design of optical communication systems at Bookham Technology (now Oclaro) and Nortel Networks, working especially on 40 Gbit WDM systems. He has also held appointments at Cambridge, Southampton and Liverpool Universities. His research interests include the quantum field theory of optical devices and all areas of optical communications, especially the theory of optical waveguide components and systems. 